\title{
BDNF Methylation and Suicidal Ideation in Patients with Acute Coronary Syndrome
}

\author{
Hee-Ju Kang1, Kyung-Yeol Bae', Sung-Wan Kim¹, Il-Seon Shin', Young Joon Hong², \\ Youngkeun Ahn' ${ }^{2}$, Myung Ho Jeong ${ }^{2}$, Jin-Sang Yoon ${ }^{1}$, and Jae-Min Kim ${ }^{1}{ }^{凶}$ \\ 1'Department of Psychiatry, Chonnam National University Medical School, Gwangju, Republic of Korea \\ ${ }^{2}$ Department of Cardiology, Chonnam National University Medical School, Gwangju, Republic of Korea
}

Objective Patients with acute coronary syndrome (ACS) are at an increased risk of suicide. It is well known that epigenetic mechanisms may explain the pathophysiology of suicidal behavior including suicidal ideation (SI), but no study has explored these mechanisms in ACS populations.

Methods In total, 969 patients were initially recruited within 2 weeks of the acute coronary event and, 711 patients were successfully followed up 1 year after ACS. SI was evaluated using the relevant items on the Montgomery-Åsberg Depression Rating Scale and covariates potentially affecting SI were estimated.

Results Brain-derived neurotrophic factor (BDNF) hypermethylation was associated with SI in both the acute and chronic phases of ACS, although the association was not statistically significant in the acute phase after applying Bonferroni's correction.

Conclusion These results suggested that BDNF hypermethylation may have played a role in an epigenetic predisposition for SI in ACS patients, particularly during the chronic phase.

Psychiatry Investig 2018;15(11):1094-1097

Key Words Acute coronary syndrome, Suicidal ideation, Brain-derived neurotrophic factor methylation, Longitudinal study.

\section{INTRODUCTION}

Acute coronary syndrome (ACS) is strongly related to a higher risk of suicide, ${ }^{1}$ and understanding the pathophysiology of suicide in ACS is important. Several biological mechanisms have been proposed to explain the pathophysiology of suicide. ${ }^{2}$ Brain-derived neurotrophic factor (BDNF) affects both neuroplasticity and neurotransmission and has been widely investigated in the context of suicide. In several studies, reduced levels of BDNF, or alterations in the BDNF allele, have been associated with suicidality. ${ }^{3,4}$ Additionally, BDNF may play an important role in atherosclerosis and the revascularization of ischemic tissue, with studies finding reduced BDNF levels in ACS patients. ${ }^{5}$ Together, these associations suggest that BDNF could affect suicidality in ACS patients.

Received: August 16,2018 Accepted: September 20,2018

$\bowtie$ Correspondence: Jae-Min Kim, MD, PhD

Department of Psychiatry, Chonnam National University Medical School, 160 Baekseo-ro, Dong-gu, Gwangju 61469, Republic of Korea

Tel: +82-62-220-6143, Fax: +82-62-225-2351

E-mail: jmkim@chonnam.ac.kr

(a) This is an Open Access article distributed under the terms of the Creative Commons Attribution Non-Commercial License (http://creativecommons.org/licenses/bync/4.0) which permits unrestricted non-commercial use, distribution, and reproduction in any medium, provided the original work is properly cited.
Epigenetic mechanisms regulate gene expression in response to environmental stimuli without changing nucleotide sequences. These mechanisms are thought to be capable of explaining complex phenomena, including suicidality. ${ }^{6}$ Epigenetic modifications of BDNF expression may provide an alternative explanation for predictors of suicide in ACS patients, as these modifications reflect interactions between BDNF gene expression and environmental stressors, such as the ACS event. Methylation of the BDNF gene has been investigated in relation to suicidal behavior in general depression, ${ }^{7,8}$ because BDNF hypermethylation was associated with reduced BDNF expression. ${ }^{8}$ However, there have been no studies evaluating the association between BDNF expression and suicidal behavior in ACS patients.

Suicidal behavior includes completed suicide, attempted suicide, and SI. SI is known to be crucial predictor of future suicide attempts and these thoughts themselves can cause a considerable burden. ${ }^{9}$ Therefore, this study focuses on SI in ACS patients. Using the data from an ACS cohort, this study aimed to investigate the longitudinal association between SI and BDNF methylation status. 


\section{METHODS}

This study was conducted as part of a prospective study of ACS patients, named the Korean DEPression in ACS (KDEPACS) study, which included a nested double-blind, randomized placebo-controlled trial: the Escitalopram for DEPression in ACS (EsDEPACS) study. The recruitment process for both studies has been described in a previous publication ${ }^{10}$ and is reiterated in Supplementary Figure 1 (in the onlineonly Data Supplement). Written informed consents and approval by the Chonnam National University Hospital Institutional Review Board were obtained.

ACS patients recently admitted to the cardiology department were approached to participate. Those who consented to both participation and blood sampling $(\mathrm{n}=969)$ comprised the acute phase sample. All acute phase participants were reapproached at 1 year to assess SI status during the chronic post-ACS phase. Of 969 patients, 711 (73\%) were successfully recruited after 1 year. There were no significant differences in characteristics between patients lost to follow-up and chronicphase participants, apart from an older mean age and higher Killip class $(\mathrm{p}<0.05)$.

SI was evaluated using the suicidal thoughts item of the Montgomery-Åsberg Depression Rating Scale (MADRS-ST). SI was evaluated within 2 weeks of the index acute coronary event, and at the 1-year follow-up. ${ }^{11}$ The presence of SI was determined by a score of 2 (fleeting suicidal thoughts) or more, as described in previous studies. ${ }^{12}$ Depression, sociodemographic, and cardiovascular characteristics potentially associated with SI in ACS patients ${ }^{13}$ were investigated as covariates within 2 weeks of the index event, as described in Supplementary Table 1 (in the online-only Data Supplement).

BDNF methylation status was determined using the PSQ 96M Pyrosequencing System (Biotage AB, Uppsala, Sweden) and Pyro Q-CpG software (ver. 1.0.9; Biotage AB) with DNA taken from leukocytes. Methylation was assessed in a cytosine-guanine $(\mathrm{CpG})$-rich area lying between -612 and -463 relative to the transcriptional start of exon VII that included nine CpG sites (Supplementary Figure 2 in the online-only Data Supplement). This region has been reported to correspond to an analogous region in rat BDNF that is differentially methylation and associated with BDNF messenger RNA expression ${ }^{14}$ and because it has been investigated in previous psychiatric studies. ${ }^{15,16} \mathrm{CpG}$ sites 2 , 4 , and 6 were excluded from the analysis as these sites showed $100 \%$ methylation in all participants. Thus, the methylation percentages at the six individual $\mathrm{CpG}$ sites, and the average value of all sites, were used in the analysis.

\section{Statistical analysis}

Demographic and clinical variables were compared according to SI status in both the acute and chronic ACS phases using t-tests or $\chi^{2}$ tests. Variables significantly associated with SI $(p<0.05)$ were included as covariates in further adjusted regression models. Analyses of the association between BDNF methylation at individual CpG sites and SI, in both the acute and chronic disease phases, were evaluated using t-tests. The independent associations between the SI and methylation status were calculated using logistic regression models after adjusting for covariates. Treatment with antidepressants was also included as a covariate in the follow-up analysis, in line with previous studies. ${ }^{13}$ Bonferroni's correction was used to maintain an overall type 1 error rate of 0.05 in the context of seven comparisons (six CpG sites and the average value of all sites). A two-sided p-value of 0.007 (0.05/7) was taken to indicate statistical significance. All analyses were conducted using SPSS software (ver. 21.0; IBM Corp., Armonk, NY, USA).

\section{RESULTS}

The BDNF methylation percentages of individual CpG sites, and the average value of all sites, were compared according to SI status at 2 weeks and 1 year after ACS (Table 1). Hypermethylation at five individual $\mathrm{CpG}$ sites (excluding CpG site 7), and the average value, were significantly associated with SI within 2 weeks of the acute coronary event. Hypermethylation of $\mathrm{CpG}$ sites 1 and 3 , and the average value, were significantly associated with SI at 1 year following ACS. After Bonferroni correction, all acute-phase associations, except between the hypermethylation of $\mathrm{CpG}$ site 3 and SI, remained statistically significant. All chronic-phase associations remained statistically significant after Bonferroni correction.

The association between BDNF methylation and SI after covariate adjustment is presented in Table 1. The statistical significance of the association between hypermethylation of CpG site 9, and the average value, with SI within 2 weeks after ACS was lost after applying the Bonferroni correction ( $\mathrm{p}=$ $0.0078-0.008$ ). However, the significant association between hypermethylation of CpG site 1 and SI at 1 year after ACS remained after correction.

\section{DISCUSSION}

This was the first study to explore longitudinal epigenetic vulnerability to SI in an ACS patient population. The data presented herein suggest that BDNF hypermethylation may predispose ACS patients to SI during both the acute and chronic phase, although the acute-phase association was not statistically significant after applying Bonferronis correction. 
Table 1. BDNF methylation status by suicidal ideation (SI) status

\begin{tabular}{|c|c|c|c|c|c|c|c|c|}
\hline \multirow{2}{*}{$\begin{array}{l}\text { Methylation } \\
\text { sites }\end{array}$} & \multicolumn{4}{|c|}{ Acute phase sample } & \multicolumn{4}{|c|}{ Follow-up sample } \\
\hline & $\begin{array}{c}\text { No SI } \\
(\mathrm{N}=774)\end{array}$ & $\begin{array}{c}\text { SI } \\
(\mathrm{N}=195)\end{array}$ & p-value & $\begin{array}{l}\text { Adjusted } \\
\text { p-value* }\end{array}$ & $\begin{array}{c}\text { No SI } \\
(\mathrm{N}=624)\end{array}$ & $\begin{array}{c}\mathrm{SI} \\
(\mathrm{N}=87)\end{array}$ & p-value & $\begin{array}{l}\text { Adjusted } \\
\text { p-value }^{\dagger}\end{array}$ \\
\hline$\overline{\mathrm{CpG}} 1$ & $14.6(2.7)$ & $15.5(2.6)$ & $<0.001^{\ddagger}$ & 0.765 & $14.8(2.8)$ & $16.0(2.6)$ & $<0.001^{\ddagger}$ & $0.006^{\ddagger}$ \\
\hline CpG 3 & $75.0(5.9)$ & $76.0(4.9)$ & 0.032 & 0.371 & $75.1(6.1)$ & $76.6(3.6)$ & $0.001^{\ddagger}$ & 0.050 \\
\hline CpG 5 & $40.6(6.5)$ & $42.0(7.0)$ & $0.006^{\ddagger}$ & 0.165 & $41.1(6.0)$ & $87.0(41.5)$ & 0.521 & 0.973 \\
\hline CpG 7 & $50.6(7.2)$ & $51.3(7.2)$ & 0.206 & 0.426 & $50.8(6.8)$ & $51.2(10.0)$ & 0.619 & 0.884 \\
\hline CpG 8 & $23.5(17.0)$ & $27.9(17.5)$ & $0.001^{\ddagger}$ & 0.082 & $23.9(16.6)$ & $26.8(17.4)$ & 0.129 & 0.369 \\
\hline CpG 9 & $20.7(20.2)$ & $26.6(21.9)$ & $<0.001^{\ddagger}$ & 0.078 & $21.1(20.1)$ & $24.6(21.3)$ & 0.135 & 0.466 \\
\hline CpG average & $37.5(5.0)$ & $39.9(5.7)$ & $<0.001^{\ddagger}$ & 0.008 & $37.8(5.2)$ & $39.5(5.0)$ & $0.004^{\ddagger}$ & 0.093 \\
\hline
\end{tabular}

Data are represented as mean (SD) unless otherwise indicated. p-values were determined using t-tests. *adjusted for gender, education, housing, current employment, previous history of depression, DSM-IV depression, tadjusted for gender, family history of depression, DSM-IV depression, treatment status (escitalopram, placebo, and medical treatment only), `statistical significance after Bonferroni’s correction

BDNF is important for neuroplasticity and neurotransmission, and hypermethylation has been associated with downregulation of BDNF expression. ${ }^{8}$ The BDNF hypermethylation seen in this study may have resulted in decreased BDNF expression, impairing neural maintenance and neural plasticity. In turn, this may have led to a reduced ability to adapt to stressful situations and an increased risk of SI in ACS patients. Additionally, BDNF has been shown to directly interact with serotonin transportation in humans. ${ }^{17}$ A hypermethylationinduced reduction in BDNF expression was associated with a reduction in the function of serotonin. Abnormal levels of serotonin are well-known to mediate suicide risk. ${ }^{2}$ Further studies investigating the exact mechanisms by which BDNF hypermethylation is associated with SI are needed, including direct measurement of BDNF expression levels.

In this study, BDNF hypermethylation was associated with SI at 1 year after the ACS event, but not within 2 weeks after ACS. These findings reflect the fact that SI in ACS patients has a number of possible etiologies, where these vary according to the time after ACS diagnosis. In the acute phase, overwhelmed physiological and psychological responses, including heightened inflammatory or stress responses, may drive SI. However, during the chronic phase, BDNF hypermethylation may take on a more important role. This hypothesis is supported by several previous publications that outline the determinants of SI according to the time elapsed after the ACS event. $^{13}$

This is the first prospective investigation to evaluate epigenetic influences on SI in patients following ACS. Epigenetic modification is a crucial transducer of environmental influence; ${ }^{6}$ thus, epigenetic factors may predict complex phenomena such as suicidality. This study is consistent with previous publications reporting that BDNF hypermethylation was associated with suicide in the postmortem brain, ${ }^{7}$ as well as with
SI in patients with depression and breast cancer. ${ }^{8,18}$ Although methylation status might differ by organ system, expression or methylation of BDNF in the central nervous system and blood stream are thought to be correlated due to the ability of BDNF to cross the blood-brain barrier. ${ }^{19}$ Therefore, our findings suggest that BDNF hypermethylation in the peripheral blood may be a biological marker of SI, particularly in ACS patients. There were several additional strengths and limitations to the current study that should be considered; these are further outlined in the Supplementary Materials (in the online-only Data Supplement).

In conclusion, the data presented herein show that BDNF hypermethylation measured at acute phase of ACS predicted SI at 1 year later. BDNF methylation status could be a biomarker for suicidality in ACS, although the finding should be replicated. Future studies are needed to examine whether new drug that modulates methylation status may contribute to decrease SI in ACS patients, since DNA methylation is known to be reversible by pharmacological agents. We believe that our study serve the basis for epigenetic mechanisms in the etiology of SI in ACS.

\section{Supplementary Materials}

The online-only Data Supplement is available with this article at https://doi.org/10.30773/pi.2018.09.20.

\section{Acknowledgments}

This study was supported by a grant (BCRI18018) Chonnam National University Hospital Biomedical Research Institute and by a grant of the National Research Foundation of Korea Grant (NRF-2017M3A9E8023015).

\section{REFERENCES}

1. Larsen KK, Agerbo E, Christensen B, Søndergaard J, Vestergaard M. Myocardial infarction and risk of suicide: a population-based casecontrol study. Circulation 2010;122:2388-2393.

2. Oquendo MA, Sullivan GM, Sudol K, Baca-Garcia E, Stanley BH, 
Sublette ME, et al. Toward a biosignature for suicide. Am J Psychiatry 2014;171:1259-1277

3. Eisen RB, Perera S, Banfield L, Anglin R, Minuzzi L, Samaan Z. Association between BDNF levels and suicidal behaviour: a systematic review and meta-analysis. Syst Rev 2015;4:187.

4. Mirkovic B, Laurent C, Podlipski MA, Frebourg T, Cohen D, Gerardin P. Genetic association studies of suicidal behavior: a review of the past 10 years, progress, limitations, and future directions. Front Psychiatry 2016;7:158.

5. Manni L, Nikolova V, Vyagova D, Chaldakov GN, Aloe L. Reduced plasma level of NGF and BDNF in patients with acute coronary syndrome. Int J Cardiol 2015;102:169-171.

6. Lockwood LE, Su S, Youssef NA. The role of epigenetics in depression and suicide: a platform for gene-environment interactions. Psychiatr Res 2015;228:235-242.

7. Keller S, Sarchiapone M, Zarrilli F, Videtic A, Ferraro A, Carli V, et al. Increased BDNF promoter methylation in the Wernicke area of suicide subjects. Arch Gen Psychiatry 2010;67:258-267.

8. Roy B, Shelton RC, Dwivedi Y. DNA methylation and expression of stress related genes in PBMC of MDD patients with and without serious suicidal ideation. J Psychiatr Res 2017;89:115-124.

9. van Spijker BA, van Straten A, Kerkhof AJ, Hoeymans N, Smit F. Disability weights for suicidal thoughts and non-fatal suicidal attempts. J Affect Disord 2011;134:341-347.

10. Kim JM, Bae KY, Kang HJ, Kim SW, Shin IS, Hong YJ, et al. Design and methodology for the Korean observational and escitalopram treatment studies of depression in acute coronary syndrome: K-DEPACS and EsDEPACS. Psychiatry Investig 2014;11:89-94.

11. Montgomery SA, Asberg M. A new depression scale designed to be sensitive to change. Br J Psychiatry 1979;134:382-389.

12. Murrough JW, Soleimani L, DeWilde KE, Collins KA, Lapidus KA, Iacoviello BM, et al. Ketamine for rapid reduction of suicidal ideation: a randomized controlled trial. Psychol Med 2015;45:3571-3580.

13. Kim JM, Kang HJ, Bae KY, Kim SW, Shin IS, Hong YJ, et al. Determinants and escitalopram treatment effects on suicidal ideation in patients with acute coronary syndrome: Findings from the K-DEPACS and EsDEPACS studies. Int J Cardiol 2016;219:225-230.

14. Roth TL, Lubin FD, Funk AJ, Sweatt JD. Lasting epigenetic influence of early-life adversity on the BDNF gene. Biol Psychiatry 2009;65:760769.

15. Devlin AM, Brain U, Austin J, Oberlander TF. Prenatal exposure to maternal depressed mood and the MTHFR C677T variant affect SLC6A4 methylation in infants at birth. PLoS One 2010;5:e12201.

16. Kim JM, Kang HJ, Bae KY, Kim SW, Shin IS, Kim HR, et al. Association of BDNF promoter methylation and genotype with suicidal ideation in elderly Koreans. Am J Geriatr Psychiatry 2014;22:989-996.

17. Henningsson S, Borg J, Lundberg J, Bah J, Lindström M, Ryding E, et al. Genetic variation in brain-derived neurotrophic factor is associated with serotonin transporter but not serotonin-1A receptor availability in men. Biol Psychiatry 2009;66:477-485.

18. Kim JM, Kang HJ, Kim SY, Kim SW, Shin IS, Kim HR, et al. BDNF promoter methylation associated with suicidal ideation in patients with breast cancer. Int J Psychiatry Med 2015;49:75-94.

19. Karege F, Vaudan G, Schwald M, Perroud N, La Harpe R. Neurotrophin levels in postmortem brains of suicide victims and the effects of antemortem diagnosis and psychotropic drugs. Brain Res Mol Brain Res 2005;136:29-37. 


\section{SUPPLEMENTARY MATERIALS}

\section{Strengths and Limitations}

There are several important considerations when interpreting the present findings. First, SI was evaluated according to the suicide-related items on the MADRS, rather than via an instrument assessing SI separately from depressed mood. Nevertheless, estimation of SI from the MADRS is a well-validated approach that has been used in previous studies. ${ }^{1}$ Second, the dependent outcome of these analyses was SI, and not suicidal behavior. The majority of previous epigenetic studies on suicide have been performed in patients who have attempted or completed suicide. ${ }^{2}$ However, SI is known to be a predictor of more severe suicidal behavior, including future suicide attempts. ${ }^{3}$ Moreover, irrespective of the presence of a suicide attempt, the inherent burden of SI is considerable. ${ }^{4}$ For these reasons, it is logical to investigate the association between BDNF methylation and SI in ACS patients, but it is difficult to generalize these findings to suicidal behavior overall. Third, patients lost to follow-up were older and had worse cardiac function in this study. Death from suicide was not recorded in those lost to follow-up. These disadvantages may contribute to the results of the follow-up analyses. Finally, the present study investigated a limited number of CpG sites in BDNF exon VI, without measuring of the expression level of BDNF. This limitation may have skewed associations toward null findings, masking differences between the groups and rendering it difficult to ascertain both the functional effects of BDNF methylation and any change in the longitudinal associations between BDNF methylation and SI.

The present study also had several strengths. It was the first prospective investigation to evaluate the epigenetic factors associated with SI in ACS patients; moreover, SI and other covariates associated with SI were assessed at similar time points (within 2 weeks and 1 year after ACS) and in a large number of patients. This reduced the potential for heterogeneity associated with differences in assessment time after ACS. Finally, participants were enrolled successively from a pool of eligible patients, all of whom were hospitalized for treatment of recent ACS. This reduced the probability of selection bias and increased the generalizability of the findings.

\section{REFERENCES}

1. Mrrough JW, Soleimani L, DeWilde KE, Collins KA, Lapidus KA, Iacoviello BM, et al. Ketamine for rapid reduction of suicidal ideation: a randomized controlled trial. Psychol Med 2015;45:3571-3580.

2. Keller S, Sarchiapone M, Zarrilli F, Videtic A, Ferraro A, Carli V, et al. Increased BDNF promoter methylation in the Wernicke area of suicide subjects. Arch Gen Psychiatry 2010;67:258-267.

3. Posner K, Brown GK, Stanley B, Brent DA, Yershova KV, Oquendo MA, et al. The Columbia-suicide severity rating scale: initial validity and internal consistency findings from three multisite studies with adolescents and adults. Am J Psychiatry 2011;168:1266-1277.

4. van Spijker BA, van Straten A, Kerkhof AJ, Hoeymans N, Smit F. Disability weights for suicidal thoughts and non-fatal suicidal attempts. J Affect Disord 2011;134:341-347. 
Supplementary Table 1. Baseline sociodemographic and clinical characteristics by suicidal ideation status

\begin{tabular}{|c|c|c|c|c|c|c|}
\hline & \multicolumn{3}{|c|}{ Baseline sample $(\mathrm{N}=969)$} & \multicolumn{3}{|c|}{ Follow-up sample $(\mathrm{N}=711)$} \\
\hline & $\begin{array}{c}\text { No suicidal } \\
\text { ideation } \\
(\mathrm{N}=774)\end{array}$ & $\begin{array}{l}\text { Suicidal } \\
\text { ideation } \\
(\mathrm{N}=195)\end{array}$ & p-value ${ }^{\dagger}$ & $\begin{array}{c}\text { No suicidal } \\
\text { ideation } \\
(\mathrm{N}=624)\end{array}$ & $\begin{array}{c}\text { Suicidal } \\
\text { ideation } \\
(\mathrm{N}=87)\end{array}$ & p-value ${ }^{\dagger}$ \\
\hline \multicolumn{7}{|l|}{ Socio-demographic characteristics } \\
\hline Age, mean (SD) years & $58.0(11.3)$ & $58.9(10.6)$ & 0.315 & $57.6(10.7)$ & $57.4(11.1)$ & 0.855 \\
\hline Sex, N (\%) female & $201(26.0)$ & $68(34.9)$ & $0.013^{*}$ & $163(26.1)$ & $33(37.9)$ & $0.021^{*}$ \\
\hline Education, mean $(\mathrm{SD})$ year & $10.0(4.7)$ & $9.1(4.4)$ & $0.012^{*}$ & $10.0(4.6)$ & $9.2(4.8)$ & 0.118 \\
\hline Living alone, $\mathrm{N}(\%)$ yes & $71(9.2)$ & $21(10.8)$ & 0.497 & 49 (7.9) & $11(12.6)$ & 0.132 \\
\hline Housing, $\mathrm{N}(\%)$ rented & $109(14.1)$ & $41(21.0)$ & $0.017^{*}$ & $102(16.3)$ & $20(23.0)$ & 0.124 \\
\hline Currently unemployed, N (\%) & $279(36.0)$ & $89(45.6)$ & $0.014^{*}$ & $213(34.1)$ & $38(43.7)$ & 0.081 \\
\hline \multicolumn{7}{|l|}{ Depression characteristics, N (\%) } \\
\hline Previous depression & $19(2.5)$ & $15(7.7)$ & $<0.001^{*}$ & $24(3.8)$ & $6(6.9)$ & 0.247 \\
\hline Family history of depression & $15(1.9)$ & $8(4.1)$ & 0.108 & $14(2.2)$ & $6(6.9)$ & $0.026^{*}$ \\
\hline DSM-IV depression & $221(28.6)$ & $157(80.5)$ & $<0.001^{*}$ & $232(37.2)$ & $53(60.9)$ & $<0.001^{*}$ \\
\hline \multicolumn{7}{|l|}{ Cardiac risk factors, $\mathrm{N}(\%)$} \\
\hline Previous ACS & $30(3.9)$ & $9(4.6)$ & 0.639 & $25(4.0)$ & $6(6.9)$ & 0.255 \\
\hline Family history of ACS & $24(3.1)$ & $7(3.6)$ & 0.729 & $18(2.9)$ & $6(6.9)$ & 0.103 \\
\hline Hypertension & $360(46.5)$ & $98(50.3)$ & 0.349 & $282(45.2)$ & $42(48.3)$ & 0.588 \\
\hline Diabetes mellitus & $144(18.6)$ & $47(24.1)$ & 0.085 & $118(18.9)$ & $24(27.6)$ & 0.058 \\
\hline Hypercholesterolemia & $384(49.6)$ & $102(52.3)$ & 0.501 & $333(53.4)$ & $50(57.5)$ & 0.472 \\
\hline Obesity & $341(44.1)$ & $74(37.9)$ & 0.123 & $280(44.9)$ & $35(40.2)$ & 0.414 \\
\hline Current smoker & $297(38.4)$ & $69(35.4)$ & 0.442 & $247(39.6)$ & $33(37.9)$ & 0.768 \\
\hline \multicolumn{7}{|l|}{ Current cardiac status } \\
\hline Killip class >1, N (\%) & $132(17.1)$ & $36(18.5)$ & 0.643 & $101(16.2)$ & $13(14.9)$ & 0.767 \\
\hline LVEF, mean (SD) \% & $61.2(11.4)$ & $61.1(10.8)$ & 0.911 & $61.4(11.0)$ & $59.7(11.8)$ & 0.183 \\
\hline Heart rate, mean $(\mathrm{SD})$ beat $/ \mathrm{min}$ & $74.7(12.1)$ & $76.1(15.8)$ & 0.230 & $75.1(12.7)$ & $74.5(13.7)$ & 0.710 \\
\hline Troponin I, mean (SD) mg/dL & $9.5(15.0)$ & $11.5(14.7)$ & 0.092 & $10.2(15.7)$ & $10.9(17.4)$ & 0.724 \\
\hline CK-MB, mean (SD) mg/dL & $16.8(38.2)$ & $19.7(33.3)$ & 0.333 & $18.1(39.1)$ & $16.6(35.5)$ & 0.736 \\
\hline \multicolumn{7}{|l|}{ Intervention group, N (\%) } \\
\hline Escitalopram & & & & $82(35.3)$ & $14(26.4)$ & $0.404 / 0.001^{*}$ \\
\hline Placebo & & & & $74(31.9)$ & $21(39.6)$ & \\
\hline Non-participants & & & & $76(32.8)$ & $18(34.0)$ & \\
\hline
\end{tabular}

*statistical significance after Bonferroni’s correction, ${ }^{\dagger} \mathrm{p}$-values were determined using t-tests or $\chi^{2}$ tests as appropriate. HAMD: Hamilton Depression Rating Scale, ACS: acute coronary syndrome, LVEF: left ventricular ejection fraction, CK-MB: Creatine kinase-MB 


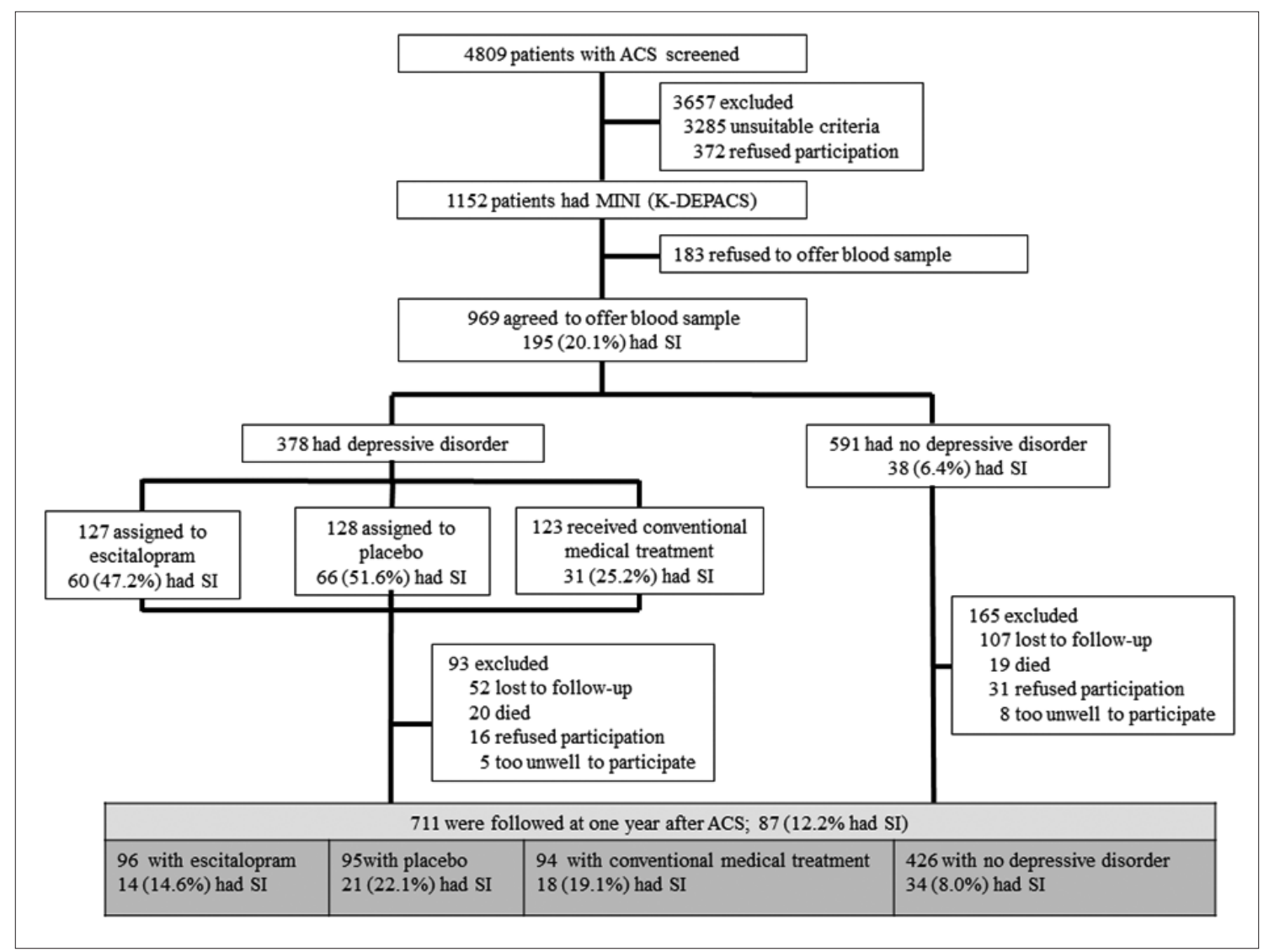

Supplementary Figure 1. Flow diagram for the recruitment process. ACS: acute coronary syndrome, MINI: Mini-International Neuropsychiatric Interview, SI: suicidal ideation, K-DEPACS: Korean DEPression in Acute Coronary Syndrome study, EsDEPACS: Escitalopram for DEPression in Acute Coronary Syndrome study. 


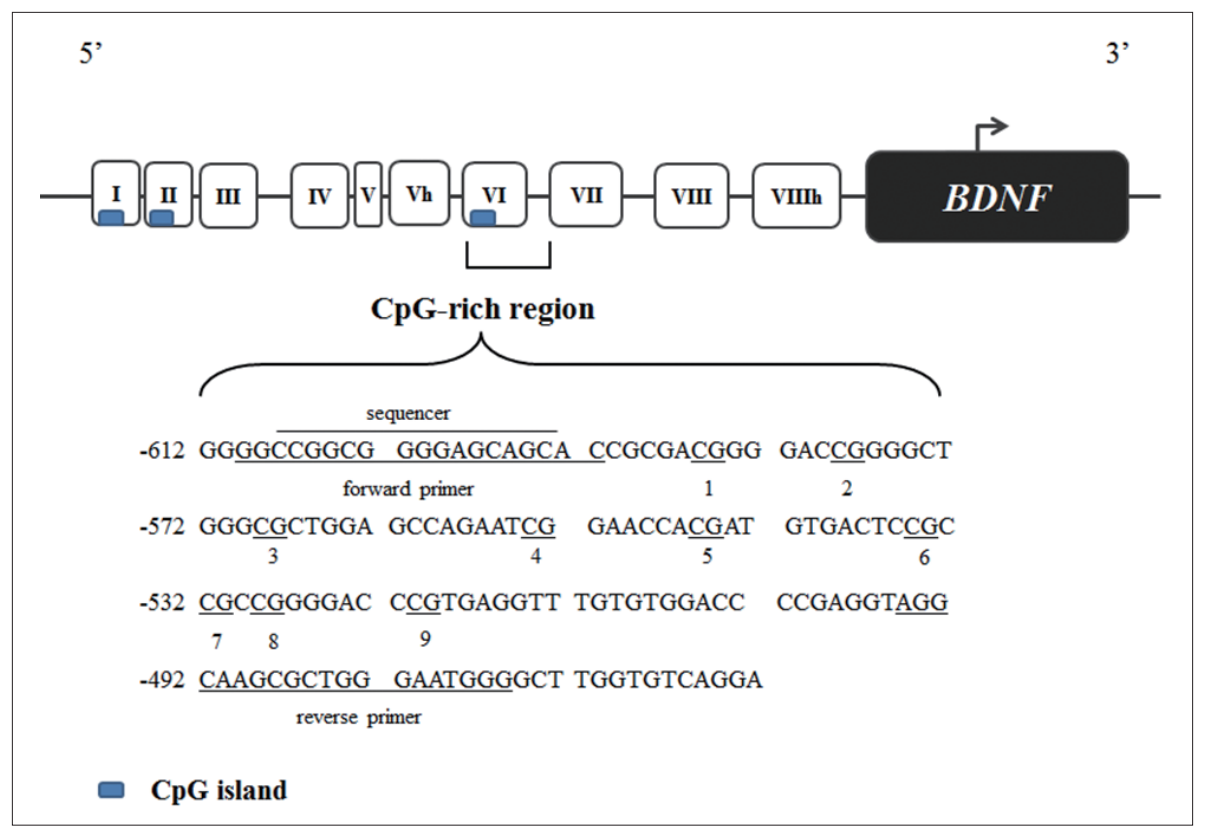

Supplementary Figure 2. Brain-derived neurotrophic factor (BDNF) exon VI cytosine-guanine (CpG) regions analyzed for methylation percentage. The $\mathrm{CpGs}$ are underlined and numbered. Forward and backward primers are shown, as well as sequencers. The genetic sequence is calculated from the transcriptional start site. CpG islands were determined as sequences of at least 200 pairs of bases with a GC percentage greater than $50 \%$. 